\title{
EPA clears the way for release of nitrogen-fixing microbe
}

\section{Washington}

LAST week, the US Environmental Protection Agency (EPA) cleared the way for the environmental release of a bacterium with an enhanced capability to fix nitrogen. The EPA proposes to permit BioTechnica International, a biotechnology company with US headquarters in Cambridge, Massachusetts, to field-test its recombinant version of Rhizobium mililoti on a five-acre plot of alfalfa in Pepin County, Wisconsin. The proposal is original in that it represents the first bacteria to be reviewed under regulations first drafted to govern toxic substances.

BioTechnica's recombinant strain of Rhizobium mililoti contains plasmids bearing multiple copies of the gene for the production of the enzyme nitrogenase, a trait that amplifies its ability to fix nitrogen. In greenhouse tests on alfalfa, increases in yield of 15-17 per cent are reported.

Farmers have used commercial preparations of the wild-type bacteria for decades to coat seeds of legumes before sowing to boost crop yields. BioTechnica hopes its recombinant bacteria will make significant inroads into this seed-coat market, estimated to be $\$ 400$ million a year for alfalfa and soybean alone.

Two other gene-spliced bacteria - a Pseudomonas that thwarts ice-nucleation on strawberry plants and another that discourages frost on potato leaves - have already received the go-ahead from EPA to be tested in the field. Advanced Genetic Sciences (AGS) of Oakland, California, sprayed a field of strawberry plants with its "ice-minus" bacteria three weeks ago (see Nature 326, 819; 1987), and two weeks ago the University of California (UC) at Berkeley planted seeds treated with its strain of Pseudomonas in a potato patch in northern California.

But the BioTechnica bacterium is being put through a different set of regulatory paces than either of the Californian ones, a procedure that disturbs environmental groups and those who would like to see a coherent, unified set of regulations to address specifically the products of biotechnology. The AGS and UC Berkeley bacteria were approved by EPA under the Federal Insecticide, Fungicide and Rodenticide Act (FIFRA), on the grounds that ice on plants is a "pest" and therefore bacteria that combat such ice-formation are "pesticides". According to a spokesperson for EPA, the fortified nitrogenfixing bacteria from BioTechnica "doesn't kill anything", so it fitted best under the aegis of the Toxic Substances Control Act (TSCA). Both acts are used by EPA to monitor gene-altered microbes under the
Coordinated Framework for Biotechnology announced in June 1986.

Critics of this two-pronged EPA review process claim that reviewing recombinant bacteria under TSCA is inappropriate, because, unlike chemical substances, bacteria have the ability to reproduce and spread.

Under TSCA, a company is required to file a pre-manufacture notification with EPA before making or importing a new chemical substance that is not on EPA's master list. EPA has 90 days to respond to this notification and perform a risk/benefit analysis to determine if the substance

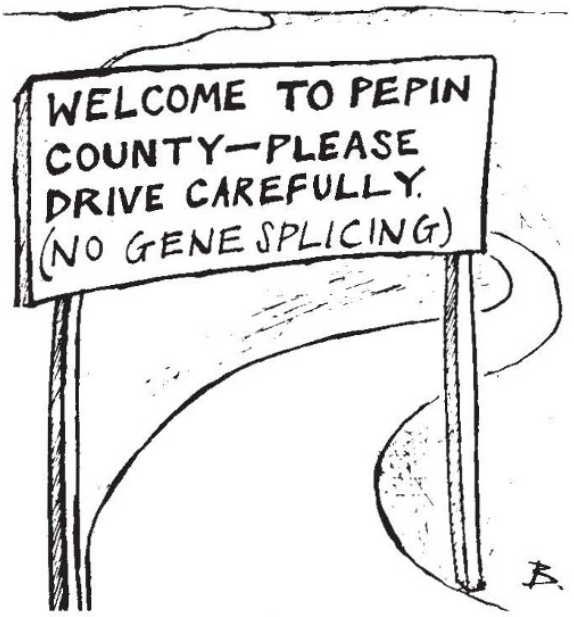

should be regulated. If a response is not forthcoming at the end of the 90 days, the company has tacit approval to make, use and sell the substance without further EPA oversight. Alternatively, EPA can extend the 90 -day response period up to an additional 90 days if more information is needed before a decision about the substance can be reached.

EPA has extended its review of the BioTechnica bacteria by 60 days to finalize the details of the field test, and allow for a brief public comment period. The risk/ benefit analysis uncovered the possibility that the enhanced nitrogen-fixing bacteria might also accelerate the growth of sweet clover, and cause it to build up coumarin, a chemical that is converted to a substance that impairs blood-clotting in animals when combined with mouldy hay. To prevent this possible side-effect, all sweet clover will be removed from the field-test plot. EPA expects to approve the field test by the end of July.

Local opposition to the field test is mobilized, however, and BioTechnica is likely to face a long haul through public forums and courthouses. Residents are circulating a petition to gather support for a one-year moratorium on the test, during which a state review and full hearings in the county would take place. Carol Ezzell
Supernova 1987a close to peak brightness

\section{Washington}

SUPERNOVA $1987 \mathrm{a}$, now with a visual magnitude of about 3.1 , may be close to the peak of its optical brightness. Recent observations show that the rate of brightening has slowed down, and at some wavelengths the expanding supernova shell may have begun to dim. But in ultraviolet radiation the brightness is still increasing, and new spectral features are beginning to emerge.

George Sonneborn of Goddard Space Flight Center and Robert Kirshner of Harvard University have been monitoring data from the International Ultraviolet Explorer (IUE) satellite since the supernova was first spotted. For several weeks there has been a steady increase in ultraviolet brightness of about 4 per cent a day, and in the past few days this has been accompanied by the appearance of rather broad lines in the ultraviolet spectrum. With the optical observations, this suggests that the supernova shell is becoming transparent, so that higher energy radiation from within can be seen directly

These new developments are more or less as expected. Everyone now agrees that the blue supergiant Sk-69 202 really was the progenitor (IUE spectra indicate that only stars 2 and 3 of the original triplet remain), and unpublished calculations by Hillebrandt et al., by Arnett and by Woosley et al., show that the explosion of such a star can produce a 'dim' Type II supernova. In addition, enough ${ }^{5 /} \mathrm{Ni}$ can be synthesized for radioactive decay to ${ }^{56} \mathrm{Co}$ and then ${ }^{56} \mathrm{Fe}$ to be the cause of the now observed ultraviolet emission.

As the shell continues to expand and rarefy, astronomers may eventually be able to see characteristic gamma rays from the radioactivity within. But the half-life for ${ }^{56} \mathrm{Co}$ to decay to stable ${ }^{56} \mathrm{Fe}$ is about 80 days, so unless the shell becomes transparent quickly, the source of gamma radiation may have expired before it becomes visible. This is one of the ways in which the evolution of the light curve can reveal the internal structure and dynamics of the supernova shell.

An unexplained aspect of the ultraviolet observations is the presence of rather broad spectral features at a number of wavelengths. Sonneborn believes they are due to absorption in the still optically thick shell, and are not emission lines from the interior, but as higher energy photons emerge from the increasingly transparent supernova remnant it should be possible to identify these features more convincingly.

David Lindley 